\title{
TRANSIENT SOLUTIONS FOR A CLASS OF DIFFRACTION PROBLEMS*
}

\author{
$\mathrm{BY}$ \\ L. B. FELSEN \\ Department of Electrophysics, Polytechnic Institute of Brooklyn
}

\begin{abstract}
The study of the fields excited by impulsive sources in layered media has been facilitated by a technique employed originally by Cagniard and Pekeris, and simplified subsequently by de Hoop. The procedure involves a reformulation of the time-harmonic solution so as to permit the explicit recovery of the transient result by inspection. In the present paper, it is shown that this method may be applied conveniently to the inversion of a certain Sommerfeld-type integral which occurs frequently in diffraction theory, thereby unifying the analysis of a class of pulse diffraction problems. Illustrative examples include the transient response to a line source in the presence of a dielectric half space, a perfectly absorbing and perfectly reflecting wedge, and a unidirectionally conducting infinite and semi-infinite screen. The latter applications illuminate the role of surface waves in the impulsive solution. It is found, in contrast to the time-harmonic case, that a different behavior characterizes the surface waves excited on a unidirectionally conducting half plane by the incident field and by the edge discontinuity, respectively.
\end{abstract}

1. Introduction. A standard procedure in the determination of the response to non-harmonic excitation is to apply the Fourier or Laplace inversion to the time-harmonic solution. $\dagger$ In diffraction problems involving unbounded regions, the steady-state response is generally given in the form of a single or double integral to which another integration is added for the recovery of the transient result. To facilitate the evaluation of the disturbance due to an impulsive source in the presence of an elastic half space, Cagniard ${ }^{5}$ proposed a method wherein the time-harmonic solution is transformed into a Laplace integral which may be inverted by inspection. The rather involved original treatment of Cagniard (see also Pekeris ${ }^{6}$ for a similar analysis) was rephrased and simplified by de Hoop ${ }^{7}$ who applied the procedure to certain elastodynamic diffraction problems and also to the determination of the fields radiated by an impulsive line or dipole source in the presence of a (non-dispersive) dielectric half-space. de Hoop's investigation, like the preceding ones, proceeds in the complex wavenumber plane (see also van der Pol and Levelt ${ }^{8}$ ), and the transformation of his time-harmonic solutions into the desired form necessitates the deformation of the original integration path away from the real axis into a hyperbolic contour in the complex plane. It is one of the purposes of the present paper to point out that the analysis is simplified further when the complex angle, rather than the complex wavenumber, plane is chosen for the representation of the steady-state results. Thus, the original integration contour is the well-known Sommerfeld path which arises in a variety of scattering problems, and the transformed contour is found to be a

*Received March 23, 1964; revised manuscript received January 2, 1965. This work was sponsored by the Air Force Cambridge Research Laboratories, Office of Aerospace Research, under Contract No. AF-19(628)-2357.

$\dagger$ For methods dealing directly with the time-dependent equations, see Keller and Blank ${ }^{1}$, Bremmer ${ }^{2}$, and Papadopoulos 3 , and the monograph by Friedlander ${ }^{4}$. 


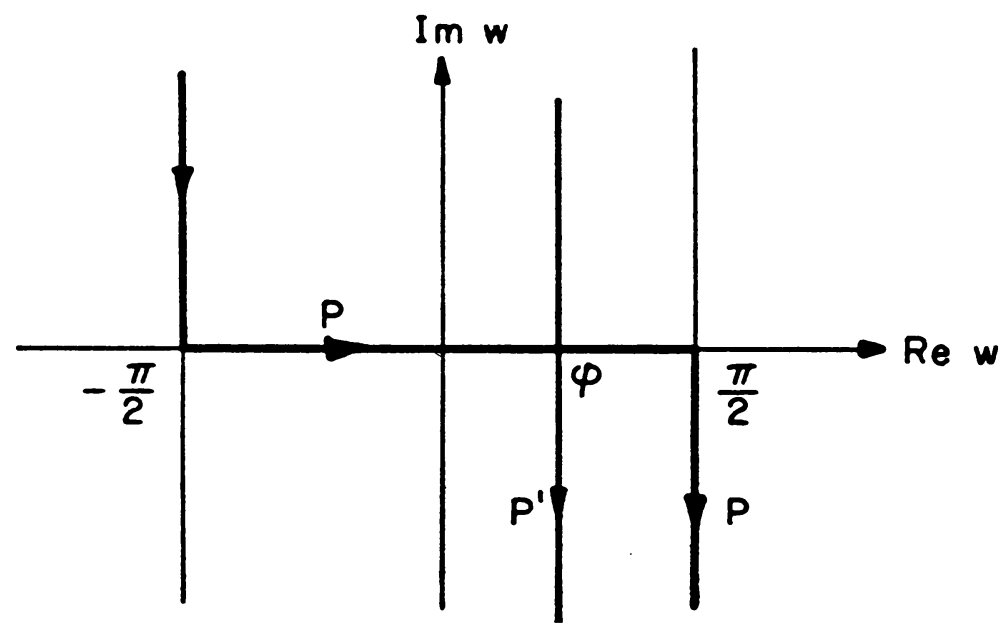

Fig. 1. Sommerfeld path for time-harmonic solution.

straight line. This facilitates the tracking of singularities which may be located between the original and the transformed paths.

Section 2 contains the steps required in the analysis, and several applications, some of which are new, are given in Section 3. It is hoped that these examples illustrate the systematization achieved in the study of a certain class of transient diffraction problems. The calculations involving a unidirectionally conducting infinite and semi-infinite plane also show the influence of surface waves at all observation times, in contrast to customary results which are valid either immediately or long after the time of arrival of the first response.

2. Transformation of the Sommerfeld integral into the desired form. Consider the integral

$$
G(\gamma, \varphi ; \omega)=a(k) \int_{P} \exp [i k \gamma \cos (w-\varphi)] u(w) d w
$$

where $w$ is the complex angle variable, and the integration path $P$ is shown in Fig. 1 . $\gamma$ is a positive real parameter, $\varphi$ is a real angle in the interval $|\varphi|<\pi / 2, k=\omega / c$ is the free-space wavenumber, $c$ is the propagation speed of light, $a(k)$ is a polynomial in $k$, and the function $u(w)$ is assumed to be independent of $k$. The solution of various steadystate diffraction problems with an implied time dependence $\exp (-i \omega t)$ may be expressed in this manner. The essence of the previously mentioned procedure ${ }^{5-7}$ is to obtain a representation in the form

$$
G(\gamma, \varphi ; i s)=b(s) \int_{0}^{\infty} \exp (-s \tau) G^{\prime}(\gamma, \varphi ; \tau) d \tau,
$$

where $\operatorname{Re} s$ is sufficiently large to assure the convergence of the integral, $b(s)$ is a real polynomial in $s$, and $G^{\prime}$ is a function of the parameters $\gamma, \varphi$, and of the real integration variable $\tau$, but not of $s$. If $\tau$ is interpreted as the temporal variable $t$, the integral in (2a) represents the one-sided Laplace transform of the time-dependent function $G^{\prime}(\gamma, \varphi ; t)$ which may therefore be recovered by inspection. The polynomial $b(s)$ corresponds to a 
time differentiation ${ }^{9}$ so that the complete expression (2a) is the Laplace transform of the function $b(d / d t) G^{\prime}(\gamma, \varphi ; t){ }^{*}$ Implicit in (2a) is a factor unity which represents the Laplace transform of the source distribution, and since $\int_{0}^{\infty} \exp (-s \tau) \delta\left(\tau-\tau^{\prime}\right) d \tau=$ $\exp \left(-s \tau^{\prime}\right)$, the corresponding temporal excitation is the impulse $\delta(t)$. Thus, if the steady-state solution $G$ can be cast into the form (2a), the transient response to a delta function impulse at $t=0$ is given by $b(d / d t) G^{\prime}(\gamma, \varphi ; t)$. For an arbitrary source function $g^{\prime}(t), t>0$, which vanishes when $t<0$, (2a) would be multiplied by the transform $g(s)=\int_{0}^{\infty} \exp (-s t) g^{\prime}(t) d t$, and the corresponding transient solution for $t>0$ is then given by superposition as

$$
g(s) G(\gamma, \varphi ; i s) \rightarrow \int_{0}^{t} g^{\prime}(\tau) b\left(\frac{d}{d t}\right) G^{\prime}(\gamma, \varphi ; t-\tau) d \tau .
$$

To achieve the formulation in (2a), one observes first that the exponential in the integrand of (1) decays inside the semi-strips

$$
0<\left(w_{r}-\varphi\right)<\pi, \quad w_{i}<0, \text { and } 0>\left(w_{r}-\varphi\right)>-\pi, \quad w_{i}>0,
$$

where $w_{r}$ and $w_{i}$ denote the real and imaginary parts of $w$. Let us assume now that $|u(w)|$ is dominated at $w_{i} \rightarrow \pm \infty$ by an exponential function so that the decay of $\exp [i k \gamma \cos (w-\varphi)]$ anywhere in the above-mentioned regions assures the convergence of the integral. If $u(w)$ has no singularities in the strip $\left|w_{r}\right|<\pi / 2$, one may deform path $P$ into the "vertical" path $P^{\prime}$ which terminates at $w=(\varphi-0)+i \infty$ and $w=$ $(\varphi+0)-i \infty$, respectively, where 0 denotes a small positive number. After a shift of the origin in the $w$-plane to $\varphi$, and the analytic continuation of the integral to positive imaginary values of $\omega$ via the introduction of the positive real variable $s=-i \omega$, one obtains

$$
G(\gamma, \varphi ; i s)=a\left(\frac{i s}{c}\right) \int_{i \infty}^{-i \infty} \exp [-s(\gamma / c) \cos w] u(w+\varphi) d w,
$$

with the integrand now decaying for $\left|w_{r}\right|<\pi / 2$. If singularities of $u(w)$ are located in the strip $\left|w_{r}\right|<\pi / 2$, these may give rise to pole or branch cut contributions which must be added to (3). The successive changes of variable $\dagger$

$$
\beta=i w, \quad \text { and } \quad \tau=\frac{\gamma}{c} \cosh \beta,
$$

then lead to the formulation

$$
G(\gamma, \varphi ; i s)=-i a\left(\frac{i s}{c}\right) \int_{\gamma / c}^{\infty}\left[\tau^{2}-\left(\frac{\gamma}{c}\right)^{2}\right]^{-1 / 2} d(\tau) \exp (-s \tau) d \tau
$$

where

$$
\begin{aligned}
d(\tau) & =u\left[\varphi-i \cosh ^{-1}\left(\frac{c \tau}{\gamma}\right)\right]+u\left[\varphi+i \cosh ^{-1}\left(\frac{c \tau}{\gamma}\right)\right] \\
& =2 \operatorname{Re} u\left[\varphi-i \cosh ^{-1}\left(\frac{c \tau}{\gamma}\right)\right], \quad \text { if } \quad u(\varphi) \quad \text { is real. }
\end{aligned}
$$

*The field behavior at $t=0$ is assumed to be such that initial value terms do not contribute.

fIf the exponential in (3) should have some other form, for example, $\exp [-s(\gamma / c) h(w)]$, the variable $\tau$ is defined as $\tau=(\gamma / c) h(w)$. 
Comparison of (2a) and (5) shows that $b(s)=-i a(i s / c)$, and

$$
G^{\prime}(\gamma, \varphi ; \tau)= \begin{cases}0, & \tau<\frac{\gamma}{c} \\ {\left[\tau^{2}-\left(\frac{\gamma}{c}\right)^{2}\right]^{-1 / 2} d(\tau),} & \tau>\frac{\gamma}{c}\end{cases}
$$

Thus, the transient solution corresponding to excitation by a temporal impulse $\delta(t)$ is given for real $u(\varphi)$ and $b(s)$ by

$$
G^{\prime}(\gamma, \varphi ; t)= \begin{cases}0, & t<\frac{\gamma}{o}, \\ -2 i a\left(\frac{i}{c} \frac{d}{d t}\right)\left\{\left[t^{2}-\left(\frac{\gamma}{c}\right)^{2}\right]^{-1 / 2} \operatorname{Re}\left(u\left[\varphi-i \cosh ^{-1}\left(\frac{c t}{\gamma}\right)\right]\right)\right\}, & t>\frac{\gamma}{c} .\end{cases}
$$

The response to an arbitrary source function $g^{\prime}(t)$, with $g^{\prime}(t) \equiv 0$ for $t<0$, may be obtained from these equations by the integration in $(2 b)$.

3. Examples. Several examples are now considered to illustrate the application of these formulas. Some of the results included are not new but their simple derivation shows the utility of the preceding analysis. In each instance, the starting point is a known time-harmonic solution involving an integral of the type shown in (1) or (3).

a. Line source in free space. The two-dimensional, time-harmonic Green's function, descriptive of radiation from a line source, is given by

$$
G_{0}(\mathfrak{\varrho} ; \omega)=\frac{i}{4} H_{0}^{(1)}(k \rho)=\frac{i}{4 \pi} \int_{P} \exp (i k \rho \cos w) d w,
$$

where $\rho$ is the radial distance from the source. Here, $a(k)=(i / 4 \pi), \gamma=\rho, u=1$, whence one finds for the time-dependent Green's function corresponding to the impulse solution in (7):

$$
G_{0}^{\prime}(\mathbf{\varrho}, t)= \begin{cases}0, & t<\frac{\rho}{c} \\ \frac{1}{2 \pi\left[t^{2}-(\rho / c)^{2}\right]^{1 / 2}}, & t>\frac{\rho}{c}\end{cases}
$$

If the line source is comprised of electric currents of unit strength, $G_{0}$ is related to the single $(x)$ component of electric field parallel to the source direction as follows:

$$
E_{x 0}(\boldsymbol{\varrho} ; \omega) e^{-i \omega t}=i \omega \mu G_{0}(\boldsymbol{\varrho} ; \omega) e^{-i \omega t}=-\mu \frac{\partial}{\partial t} G_{0}(\boldsymbol{\varrho} ; \omega) e^{-i \omega t},
$$

where $\mu$ is the free-space permeability. The time dependent field $E_{x}^{\prime}(\boldsymbol{0} ; t)$ corresponding to the impulsive excitation $\mathbf{J}(\mathbf{r}, t)=\mathbf{x}_{0} \delta(\mathbf{o}) \delta(t)$ may therefore be obtained from $G_{0}^{\prime}$ by a time differentiation. Dual considerations apply to a line of magnetic currents.

b. Line source and dielectric half space. Assume that a line source of electric current is located in the vacuum half space $z<0$ at the point $\boldsymbol{\varrho}^{\prime} \equiv\left(y^{\prime}, z^{\prime}\right)=\left(0, z^{\prime}\right), z^{\prime}<0$, and 
that the half space $z>0$ is filled with a lossless homogeneous dielectric having a relative permittivity $\epsilon$. For observation points in the vacuum region, the time harmonic Green's function (proportional to the $x$-component of the electric field as in (10) is known to be ${ }^{10}$ :

$$
G\left(\mathbf{\varrho}, \mathfrak{\varrho}^{\prime} ; \omega\right)=G_{0}\left(\mathbf{\varrho}, \mathfrak{\varrho}^{\prime} ; \omega\right)+G_{s}\left(\boldsymbol{\varrho}, \mathfrak{\varrho}^{\prime} ; \omega\right),
$$

where $G_{0}$ represents the primary contribution in (8) (with $\rho \rightarrow\left|\boldsymbol{\varrho}-\mathbf{o}^{\prime}\right|$ ) while $G_{\boldsymbol{s}}$ involves the reflection effects due to the interface:

$$
G_{s}\left(\boldsymbol{\varrho}, \boldsymbol{\varrho}^{\prime} ; \omega\right)=\frac{i}{4 \pi} \int_{P} \exp [i k R \cos (w-\varphi)] \Gamma(w) d w .
$$

$\Gamma(w)$ is the plane wave reflection coefficient

$$
\Gamma(w)=\frac{\cos w-\left(\epsilon-\sin ^{2} w\right)^{1 / 2}}{\cos w+\left(\epsilon-\sin ^{2} w\right)^{1 / 2},}
$$

with the square root positive when the radicand is positive, and $(R, \varphi)$ defined as the cylindrical polar coordinates relative to the image point,

$$
y=R \sin \varphi, \quad\left|z+z^{\prime}\right|=R \cos \varphi .
$$

Since $\epsilon>1, \Gamma(w)$ has no singularities in the region $|\operatorname{Re} w|<\pi / 2$. (The integration path is indented into the region $|\operatorname{Re} w|<\pi / 2$ to avoid the branch points at $\sin w_{b}= \pm \epsilon^{1 / 2}$ ). If the dielectric is assumed to be non-dispersive so that $\epsilon$ is independent of $\omega$, all of the conditions in Sec. 2 are satisfied and the impulse response may be recovered by inspection:

$$
G^{\prime}\left(\boldsymbol{\varrho}, \mathfrak{\varrho}^{\prime} ; t\right)=G_{0}^{\prime}\left(\left|\boldsymbol{\varrho}-\mathfrak{\varrho}^{\prime}\right| ; t\right)+G_{s}^{\prime}\left(\boldsymbol{\varrho}, \mathfrak{\varrho}^{\prime} ; t\right),
$$

where

$$
G_{s}^{\prime}\left(\mathbf{\varrho}, \mathbf{e}^{\prime} ; t\right)=\left\{\begin{array}{ll}
0, & t<\frac{R}{c} \\
\left\{2 \pi\left[t^{2}-\left(\frac{R}{c}\right)^{2}\right]^{1 / 2}\right\}^{-1} \operatorname{Re}\left\{\Gamma\left[\varphi-i \cosh ^{-1}\left(\frac{c t}{R}\right)\right]\right\}, & t>\frac{R}{c}
\end{array} .\right.
$$

The non-dispersive assumption for $\epsilon$ is not valid for a physical dielectric subjected to impulsive excitation but holds approximately for other excitation functions whose frequency spectrum is confined. The field in the vacuum half space evidently consists of two contributions, the first of which is the direct pulse arriving at $t=\left|\boldsymbol{\varrho}-\mathbf{\varrho}^{\prime}\right| / c$ while the second is the reflected pulse which arrives at $t=R / c$, appears to emanate from the image point $R=0$, and has an amplitude dependence modified by Re $\Gamma$.

If the source and observation points lie in the dielectric region, the formulation is analogous to the above except that $\epsilon$ is now a quantity smaller than unity. In this instance, $\Gamma(w)$ has branch point singularities on the real $w$-axis at $w= \pm \sin ^{-1}\left(\epsilon^{1 / 2}\right)$ and these singularities will be crossed during the path deformation in (1) if $\varphi>\sin ^{-1}\left(\epsilon^{1 / 2}\right)$. The corresponding branch cut integral contribution gives rise to additional waves (lateral waves) which are intimately connected with the phenomenon of total reflection. ${ }^{8}$

Radiation from a dipole source may also be treated by this method but the transformation of the integral is more complicated than that for the line source. ${ }^{7 b}$

c. Impulse excitation of a "perfectly absorbing" wedge. The previously described line source of electric currents is now assumed to be located at the point $\left(\rho^{\prime}, \phi^{\prime}\right)$ in the presence 


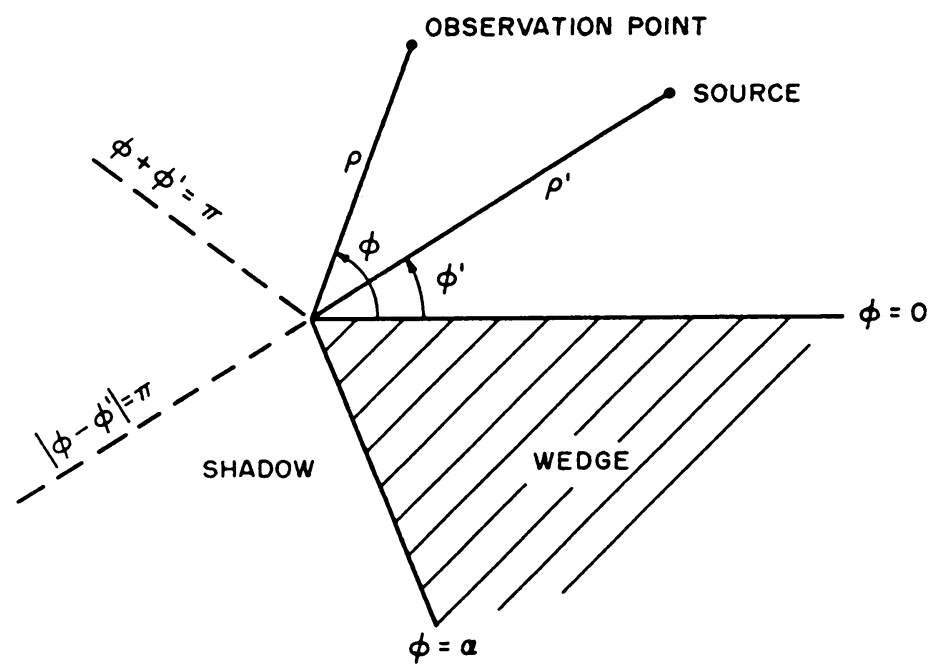

FIG. 2. Diffraction by a wedge.

of a "perfectly absorbing" wedge which occupies the exterior region bounded by the half planes $\phi=0$ and $\phi=\alpha$. Here, $(\rho, \phi)$ are cylindrical polar coordinates centered at the wedge apex. The perfectly absorbing wedge is an idealized structure which is analogous to Sommerfeld's "black screen" "and which absorbs completely all "angularly propagating" incident waves. ${ }^{12}$ This diffraction problem is the simplest which can be phrased for a wedge-shaped configuration, and its solution, while of interest in its own right as regards the study of absorbing surfaces, may be used to synthesize the result appropriate to perfectly reflecting boundaries. Since the line source problem is somewhat more involved than those for plane wave or point source excitation, the latter are considered first. For the plane wave case, the electric vector is taken parallel to the edge whereas the point source excitation is assumed to arise from an axial electric current element. The total electromagnetic fields are then easily derivable from the scalar functions $G$ discussed below.

If a plane wave $G_{0}\left(\boldsymbol{0}, \phi^{\prime} ; \omega\right)=\exp \left\{-i k \rho \cos \left(\phi-\phi^{\prime}\right)\right\}$ is incident from the direction $\phi=\phi^{\prime}$ in Fig. 2, the time harmonic solution is known to be given by ${ }^{12}$ :

$$
G\left(\boldsymbol{\varrho}, \phi^{\prime} ; \omega\right)=G_{0}\left(\mathbf{\varrho}, \phi^{\prime} ; \omega\right)+G_{s}\left(\mathbf{\varrho}, \phi^{\prime} ; \omega\right)
$$

where

$$
\begin{aligned}
& G_{s}\left(\mathbf{\varrho}, \phi^{\prime} ; \omega\right)=\frac{-i}{2 \pi} \int_{P} \exp [i k \rho \cos w] A\left(\phi, \phi^{\prime} ; w\right) d w, \\
& A\left(\phi, \phi^{\prime} ; w\right)=\frac{1}{\pi-\left|\phi-\phi^{\prime}\right|-w}+\frac{1}{\pi+\left|\phi-\phi^{\prime}\right|+w},
\end{aligned}
$$

and the integration path is indented into the lower half of the complex $w$-plane to avoid the pole at $w=\pi-\left|\phi-\phi^{\prime}\right|$. The deformed path $P^{\prime}$ in Fig. 1 now passes along the axis $\operatorname{Re} w=0$ so that the pole is crossed during the deformation when $\left|\phi-\phi^{\prime}\right|>\pi$. The resulting residue cancels exactly the contribution from $G_{0}$, thereby limiting the domain of existence of the plane wave to the illuminated region $\left|\phi-\phi^{\prime}\right|<\pi$. Diffraction effects 
are accounted for by the integral along $P^{\prime}$ from which the impulse response is recovered directly via Eqs. (7):

$$
G^{\prime}\left(\boldsymbol{\varrho}, \phi^{\prime} ; t\right)=\delta\left[t+\frac{\rho}{c} \cos \left(\phi-\phi^{\prime}\right)\right] \eta\left(\pi-\left|\phi-\phi^{\prime}\right|\right)+G_{s}^{\prime}\left(\boldsymbol{\varrho}, \phi^{\prime} ; t\right),
$$

where $\eta(x)=1$ or 0 when $x>0$ and $x<0$, respectively, and

$$
G_{s}^{\prime}\left(\boldsymbol{\varrho}, \phi^{\prime} ; t\right)= \begin{cases}0, & t<\frac{\rho}{c} \\ -\left\{\pi\left[t^{2}-\left(\frac{\rho}{c}\right)^{2}\right]^{1 / 2}\right\}^{-1} \operatorname{Re}\left\{A\left[\phi, \phi^{\prime} ; i \cosh ^{-1}(c t / \rho)\right]\right\}, & t>\frac{\rho}{c}\end{cases}
$$

Thus, the diffraction field is established at time $t=0$ when the incident pulse strikes the edge, and takes the form of a cylindrically spreading disturbance which reaches an observation point $B$ after the time interval $(\rho / c)$ required to cover the distance from the edge to $B$. Because of the "perfectly absorbing" boundary condition at the wedge surface, no reflected contribution is present.

For excitation by a point source located at $\mathbf{r}^{\prime}=\left(\rho^{\prime}, \phi^{\prime}, x^{\prime}\right)$, with $x$ denoting the coordinate along the edge, the time-harmonic primary field is

$$
\begin{aligned}
G_{0}\left(\mathbf{r}, \mathbf{r}^{\prime} ; \omega\right) & =\frac{\exp \left[i k \zeta\left(\pi-\left|\phi-\phi^{\prime}\right|\right)\right]}{4 \pi \zeta\left(\pi-\left|\phi-\phi^{\prime}\right|\right)}, \\
\zeta(w) & =\left[\rho^{2}+{\rho^{\prime}}^{2}+2 \rho \rho^{\prime} \cos w+\left(x-x^{\prime}\right)^{2}\right]^{1 / 2},
\end{aligned}
$$

and the wedge introduces the secondary potential ${ }^{12}$

$$
G_{s}\left(\mathbf{r}, \mathbf{r}^{\prime} ; \omega\right)=\frac{-i}{8 \pi^{2}} \int_{P} \frac{\exp [i k \zeta(w)]}{\zeta(w)} A\left(\boldsymbol{\phi}, \phi^{\prime} ; w\right) d w,
$$

with the integration path taken around the pole as above. The branch points of $\zeta(w)$, which is defined positive when $w=0$ and with a positive imaginary part when nonreal, lie outside the interval $|\operatorname{Re} w|<\pi / 2$ and are of no concern. After deforming into the imaginary axis (with $k=i s / c$ ), the recovery of the transient solution now involves the change of variable $\tau=\zeta(-i \beta) / c$ (see footnote p.153), and proceeds without difficulty.

The line source problem is more involved since the primary field $G_{0}\left(\boldsymbol{\varrho}, \varrho^{\prime} ; \omega\right)$ in (8) now gives rise to the secondary potential ${ }^{12}$

$$
G_{s}\left(\boldsymbol{\varrho}, \boldsymbol{\varrho}^{\prime} ; \omega\right)=\frac{1}{8 \pi} \int_{P} H_{0}^{(1)}\left[k\left(\rho^{2}+{\rho^{\prime 2}}^{2}+2 \rho \rho^{\prime} \cos w\right)^{1 / 2}\right] A\left(\phi, \phi^{\prime} ; w\right) d w,
$$

all definitions being analogous to the above. $k$ does not appear in exponential form so that some prior manipulations are required before the impulse response may be determined conveniently. Letting $k=(i s / c)$ and deforming into the imaginary axis, one obtains a residue contribution from the pole at $w=\pi-\left|\phi-\phi^{\prime}\right|$ which cancels the primary field in the shadow region $\left|\phi-\phi^{\prime}\right|>\pi$. In view of the formula,

$$
H_{\nu}^{(1)}(i z)=\frac{2}{\pi i} \exp (-i \nu \pi / 2) K_{\nu}(z),
$$

where $K_{\nu}(z)$ is the modified Bessel function, one finds for the secondary potential, 


$$
G_{s}=\frac{i}{4 \pi^{2}} \int_{-i \infty}^{i \infty} K_{0}\left[\frac{s}{c}\left(\rho^{2}+{\rho^{\prime}}^{2}+2 \rho \rho^{\prime} \cos w\right)^{1 / 2}\right] A\left(\phi, \phi^{\prime} ; w\right) d w .
$$

To make this expression correspond to the one in (3), it is suggestive to introduce

$$
K_{0}(z)=\int_{1}^{\infty}\left(\zeta^{2}-1\right)^{-1 / 2} \exp (-\zeta z) d \zeta, \quad z>0
$$

to obtain

$$
G_{s}=\frac{i}{4 \pi^{2}} \int_{-i \infty}^{i \infty} d w A\left(\phi, \phi^{\prime} ; w\right) \int_{v / c}^{\infty}\left[\tau^{2}-\left(\frac{v}{c}\right)^{2}\right]^{-1 / 2} \exp (-s \tau) d \tau,
$$

where

$$
v \equiv v(\beta)=\left[\rho^{2}+{\rho^{\prime}}^{2}+2 \rho \rho^{\prime} \cosh \beta\right]^{1 / 2}>0, \quad \beta=i w .
$$

An interchange of the orders of integration, which in (23) cover the range $v(\beta)<c \tau<\infty$ and $\infty>\beta>-\infty$, results in $f(\tau)>\beta>-f(\tau)$ and $v(0)<c \tau<\infty$, where

$$
f(\tau)=\cosh ^{-1}\left[\frac{c^{2} \tau^{2}-\rho^{2}-{\rho^{\prime}}^{2}}{2 \rho \rho^{\prime}}\right],
$$

and the $\beta$ integration is performed first. Thus,

$$
G_{s}=\int_{v(0) / c}^{\infty} d \tau \exp (-s \tau) \int_{-f(\tau)}^{f(\tau)}\left\{-4 \pi^{2}\left[\tau^{2}-\frac{v^{2}(\beta)}{c^{2}}\right]^{1 / 2}\right\}^{-1} A\left(\phi, \phi^{\prime} ;-i \beta\right) d \beta,
$$

from which expression the impulse solution is recovered directly, however in the form of an integral,

$$
G_{s}^{\prime}\left(\boldsymbol{\varrho}, \boldsymbol{\varrho}^{\prime} ; t\right)=\left\{\begin{array}{lr}
0, & t<\frac{\rho+\rho^{\prime}}{c}, \\
\int_{0}^{f(t)}\left\{-2 \pi^{2}\left[t^{2}-\frac{v^{2}(\beta)}{c^{2}}\right]^{1 / 2}\right\}^{-1} \operatorname{Re} A\left(\phi, \phi^{\prime} ;-i \beta\right) d \beta, t>\frac{\rho+\rho^{\prime}}{c} .
\end{array}\right.
$$

To this diffracted field, which arrives at the observation point after the time interval $\left(\rho+\rho^{\prime}\right) / c$ required to cover the distance from the source point to the edge and from the edge to the observation point, must be added the primary field $G_{0}^{\prime}\left(\left|\boldsymbol{e}-\boldsymbol{\varrho}^{\prime}\right| ; t\right)$ in the illuminated region.

d. Impulse excitation of a perfectly conducting wedge. If the wedge in Fig. 2 is now assumed to be perfectly conducting, the Green's function $G$ is required to vanish on the wedge surface. This boundary condition may be satisfied by superposing an infinite set of "image solutions" for the perfectly absorbing wedge, and the resulting series may be summed into a closed form. ${ }^{12}$ The following well-known expression is then obtained for the line source field in the presence of the perfectly conducting wedge ${ }^{13}$ :

$$
\begin{gathered}
G\left(\mathbf{\varrho}, \mathbf{\varrho}^{\prime} ; \omega\right)=G_{1}\left(\mathbf{\varrho}, \mathbf{\varrho}^{\prime} ; \omega\right)+G_{s}\left(\mathbf{\varrho}, \mathbf{\varrho}^{\prime} ; \omega\right), \\
G_{1}\left(\mathbf{\varrho}, \mathbf{\varrho}^{\prime} ; \omega\right)=\frac{i}{4} H_{0}^{(1)}\left[k R\left(\phi-\phi^{\prime}\right)\right] \eta\left[\pi-\left|\phi-\phi^{\prime}\right|\right]-\frac{i}{4} H_{0}^{(1)}\left[k R\left(\phi+\phi^{\prime}\right)\right] \eta\left[\pi-\left(\phi+\phi^{\prime}\right)\right] \\
-\frac{i}{4} H_{0}^{(1)}\left[k R\left(2 \alpha-\phi-\phi^{\prime}\right)\right] \eta\left[\pi-\left(2 \alpha-\phi-\phi^{\prime}\right)\right], \\
R(x)=\left[\boldsymbol{\rho}^{2}+{\boldsymbol{\rho}^{\prime 2}}^{2}-2 \rho \rho^{\prime} \cos x\right]^{1 / 2},
\end{gathered}
$$


where it is assumed that the exterior wedge angle $\alpha$ is greater than $\pi$, and $G_{1}$ contains the primary field $G_{0}$ as well as the geometrically reflected field contributions. The diffracted field $G_{s}$ is still given by $(21)$ provided that $A\left(\phi, \phi^{\prime} ; w\right)$ is taken as the function

$$
\begin{gathered}
A\left(\phi, \phi^{\prime} ; w\right)=A_{-}-A_{+}, \\
A_{ \pm}=\frac{\pi}{\alpha} \frac{\sin [(\pi / \alpha)(w-\pi)]}{\cos [(\pi / \alpha)(w-\pi)]-\cos \left[(\pi / \alpha)\left(\phi \pm \phi^{\prime}\right)\right]} .
\end{gathered}
$$

The pulse solution may therefore be recovered by a direct utilization of the expressions given in the preceding section, and contains reflected waves in addition to the primary and diffracted fields. ${ }^{13}$ If the wedge degenerates into a half plane $(\alpha=2 \pi)$, the diffracted wave integral (25b) may be evaluated (reference 13, Sec. 6.2 ; ref. 4 , p. 126) so that line source, point source and plane wave excitation yield simple expressions for the fields.

e. Line source and unidirectionally conducting screen. An interesting class of electromagnetic boundary value problems involves "unidirectionally" conducting surfaces on which the flow of induced electric currents is constrained to a specified direction. If the preferred direction is parallel to a rectilinear coordinate, say $y^{\prime}$, the boundary conditions on such a surface require the vanishing of the electric field component parallel to $y^{\prime}$, while the parallel component of the magnetic field and the perpendicular components of the electric field are continuous through the surface. Thus, for an infinite, plane, unidirectionally conducting screen confined to the $z=0$ plane,

$$
\begin{gathered}
E_{y^{\prime}}=0 \text { at } z=0, \\
H_{y^{\prime}} \text { and } E_{x^{\prime}} \text { continuous at } z=0 .
\end{gathered}
$$

Such a surface may be realized approximately by a layer of straight, insulated, tightly packed conducting filaments provided that the wavelength of the incident excitation is large compared with the cross-sectional dimension of each element. The fields excited by an arbitrarily oriented line source of electric currents parallel to the surface have been calculated by Seshadri ${ }^{14}$, and his solution is employed herein to derive the transient response to a temporal impulse. In this application, the boundary conditions $(27 \mathrm{a}, \mathrm{b})$ are assumed to hold at all frequencies, thereby casting doubt upon the physical realizability of this configuration; however, if the impulse solution is employed to synthesize the fields due to a transient source with a confined frequency spectrum, the non-dispersive characteristics in $(27 \mathrm{a}, \mathrm{b})$ may be approximated by a suitable physical structure. While the results in Secs. 3a, b, d are available in the technical literature, though by alternative derivations, the transient fields in the presence of a unidirectionally conducting screen have not been evaluated previously. This problem is especially interesting since it illuminates in a simple manner the role played by surface waves in the buildup of the field response.

The physical configuration is shown in Fig. 3, where the line source extends parallel to the $x$-axis and is located at the point $\left(0, z^{\prime}\right), z^{\prime}<0 ; \alpha$ is the angle between the positive $x$-axis and the positive $y^{\prime}$-axis defining the direction of conductivity on the screen. The field components excited by a line current oscillating according to a time dependence $\exp (-i \omega t)$ have been given by Seshadri and are conveniently separated into a primary field (see (8) and (10) and a secondary field which accounts for the presence of the screen. The secondary fields $\mathbf{E}_{1}$ and $\mathbf{H}_{1}$ are expressed in integral form, and since all the components may be derived by suitable differentiations from $E_{\mathrm{x} 1}=$ 

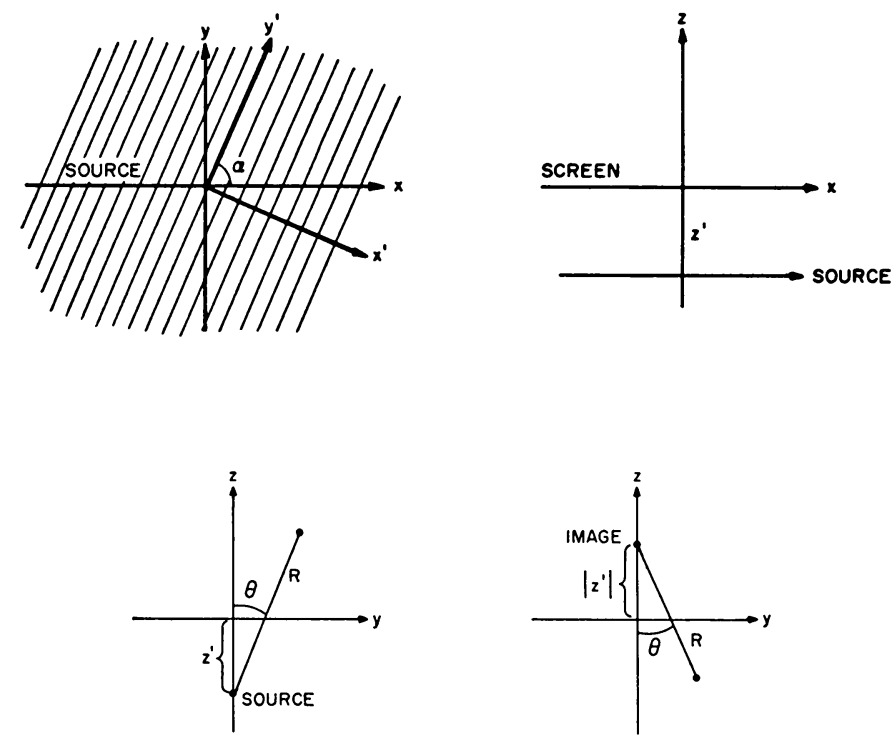

$z>0$

$z<0$

Fig. 3. Physical configuration (unidirectionally conducting screen).
a) Top view
b) Side view
c) End view

$\left.-\mu(\partial / \partial t) G_{1} \mathbf{\varrho}, \mathbf{\varrho}^{\prime} ; \omega\right) e^{-i \omega t}$, this component alone is considered further. In the notation of the present paper, Seshadri's expression is (reference 14, Eqs. (23)-(25)):

$$
G_{1}\left(\boldsymbol{\varrho}, \boldsymbol{\varrho}^{\prime} ; \omega\right)=\frac{-i \cot ^{2} \alpha}{4 \pi} \int_{P} \frac{\exp [i k R \cos (w-\theta)]}{\csc ^{2} \alpha-\sin ^{2} w} d w, \quad \mathbf{\varrho}=(y, z),
$$

where the observation point coordinates $(R, \theta)$ are defined with respect to the image point when $z<0$ and with respect to the source point when $z>0$ (see Fig. 3(c); because of symmetry it suffices to consider the region $y>0$ ):

$$
y=R \sin \theta, \quad|z|+\left|z^{\prime}\right|=R \cos \theta, \quad 0 \leq \theta \leq \pi / 2,
$$

and $P$, the integration path shown in Fig. 1, is indented into the strip $\left|w_{r}\right|<\pi / 2$ to avoid the pole singularities at $w_{p}= \pm\left(\pi / 2-i \cosh ^{-1} \csc \alpha\right){ }^{*}$ These poles give rise to surface waves whose influence on the time-harmonic field is expressed explicitly in the alternative representation obtained by shifting the integration path to the right through an interval $\pi / 2$ and then defining $(w-\pi / 2)$ as the new variable:

$$
\begin{aligned}
G_{1}\left(\boldsymbol{\varrho}, \boldsymbol{\varrho}^{\prime} ; \omega\right)=\frac{i \cos \alpha}{4 \pi} \exp [i k y \csc \alpha & -k Z \cot \alpha] \\
& -\frac{i \cot ^{2} \alpha}{4 \pi} \int_{P} \frac{\exp [i k R \cos (w-\varphi)]}{\cot ^{2} \alpha+\sin ^{2} w} d w,
\end{aligned}
$$

where $\varphi=\pi / 2-\theta$ and $Z=|z|+\left|z^{\prime}\right|$. The first term in (28b) represents a surface wave which decays exponentially in the perpendicular direction on both sides of the screen and which propagates along the $y$-direction with the frequency independent phase

*Other poles are present at $w_{p}= \pm\left(\pi / 2+i \cosh ^{-1} \csc \alpha\right)$. 
velocity $v=c \sin \alpha$, slower than the propagation speed $c$ of light in empty space. In the representation (28b), the integral now has poles on the imaginary axis at $w_{p}= \pm i$ $\sinh ^{-1} \cot \alpha$. For observation points near the surface, an asymptotic evaluation of the integral for large values of $R$ yields only a reflected space wave contribution which is cancelled to $0\left\{(k R)^{-1 / 2}\right\}$ by the incident field, thereby leaving the surface wave predominant. ${ }^{14}$

The response to an impulsive excitation $\delta(t)$ is recovered at once upon comparing (28) with (1) and (7a, b):

$$
G_{1}^{\prime}\left(\mathbf{o}, \mathbf{o}^{\prime} ; t\right)= \begin{cases}0, & t<\frac{R}{c}, \\ -\cos ^{2} \alpha\left\{2 \pi\left[t^{2}-\left(\frac{R}{c}\right)^{2}\right]^{1 / 2}\right\}^{-1} & \\ \cdot \operatorname{Re}\left\{\frac{1}{1-\sin ^{2} \alpha \sin ^{2}\left[\theta-i \cosh ^{-1}(c t / R)\right]}\right\}, & t>\frac{R}{c} .\end{cases}
$$

The same result is obtained from (28b) after the recognition that the transition from (1) to (3) requires in this instance the extraction of a pole which cancels exactly the first term in (28b). Thus, the transient solution contains no explicit evidence of the surface waves which play such an important role in the time-harmonic problem. The surface waves do, however, influence the transient response as shown by the investigation below. Before proceeding further, we exhibit the explicit form of the numerator term in $(29 b)$,

$$
\operatorname{Re}\{\}=\frac{\csc ^{2} \alpha-\cos ^{2} \theta+(\cos 2 \theta)(c t / R)^{2}}{\left[\csc ^{2} \alpha-\cos ^{2} \theta+(\cos 2 \theta)(c t / R)^{2}\right]^{2}+\left(\sin ^{2} 2 \theta\right)(c t / R)^{2}\left[(c t / R)^{2}-1\right]} \csc ^{2} \alpha,
$$

whence the detailed space-time behavior of the secondary field is not excessively complicated. To this contribution must be added the incident field,

$$
G_{0}^{\prime}\left(\mathbf{\varrho}, \mathfrak{\varrho}^{\prime} ; t\right)= \begin{cases}0, & t<\frac{\left|\boldsymbol{\varrho}-\mathbf{Q}^{\prime}\right|}{c}, \\ \frac{1}{2 \pi} \overline{\left[t^{2}-\left(\left|\boldsymbol{\varrho}-\mathfrak{\varrho}^{\prime}\right|^{2} / c^{2}\right)\right]^{1 / 2}}, & t>\frac{\left|\boldsymbol{\varrho}-\mathbf{\varrho}^{\prime}\right|}{c} .\end{cases}
$$

One observes from (29b) that the first secondary arrival is at $t=R / c$, the time required for the pulse to travel via a geometric-optical path from the source to the screen and from the screen to the observation point. For $t \approx R / c$, the numerator in $(29 \mathrm{~b})$ introduces little distortion so that the initial reflected field is essentially that of a simple line source at $R=0$, modified by the angle-dependent reflection factor $-\cot ^{2} \alpha\left\{\csc ^{2} \alpha-\sin ^{2} \theta\right\}^{-1}$. It is noted, incidentally, that the secondary potential $G_{1}^{\prime}$ reduces to 0 when $\alpha=\pi / 2$ and to $-G_{0}^{\prime}\left(\mathbf{\varrho}, \mathfrak{\varrho}_{1}^{\prime} ; t\right)$ when $\alpha=0$, where $\boldsymbol{\varrho}_{1}^{\prime}$ is the coordinate of the point $R=0$. This behavior describes correctly the limiting cases where the screen is, respectively, perfectly transparent and perfectly reflecting.

It is worthwhile to consider explicitly two special cases for which substantial simplification occurs in (29c): $\theta=0$ (observation points in the plane perpendicular to the screen and containing the source), and $\theta=\pi / 2$ (source and observation points on the screen). In the first instance, for $t>R / c$, 


$$
G_{1}^{\prime}\left(\mathbf{o}, \mathbf{o}^{\prime} ; t\right)=-\left\{2 \pi\left[1+\left(\tan ^{2} \alpha\right)\left(\frac{c t}{R}\right)^{2}\right]\left[t^{2}-\left(\frac{R}{c}\right)^{2}\right]^{1 / 2}\right\}^{-1}, \quad \theta=0,
$$

so that the reflected potential has a monotonic time behavior which is not unlike that for a perfect conductor but is accentuated by the presence of the unidirectional conductivity on the screen. The situation is quite different in the second case where one may conveniently combine the incident and secondary contributions (since $\left|\boldsymbol{\varrho}-\boldsymbol{\varrho}^{\prime}\right|=$ $R=y):^{*}$

$$
G^{\prime}\left(\mathbf{o}, \mathbf{o}^{\prime} ; t\right)=\frac{1}{2 \pi} \frac{\left[t^{2}-(y / c)^{2}\right]^{1 / 2}}{t^{2}-\left(\csc ^{2} \alpha\right)(y / c)^{2}}, \quad \theta=\frac{\pi}{2}, \quad t>\frac{y}{c},
$$

with $G^{\prime}=0$ for $t<y / c$. The first response at $y$ occurs again at $t=y / c$, after a time interval required by a signal traveling at the speed of light to cover the distance between the source and observation points. A sharp increase is observed at a later time $t=(y / c)$ csc $\alpha$, corresponding to the signal velocity $v=c \sin \alpha$ associated with the previously discussed surface waves in the time-harmonic problem (Fig. 6(a)). Thus, the existence of surface waves on the unidirectionally conducting screen gives rise to a peak in the response function which (for $\theta=\pi / 2$ ) occurs precisely after the time required for these waves to arrive at the observation point (on this non-dispersive surface, all surface waves travel with the same speed). The height of the peak is infinite when the source and observation points both lie on the surface but diminishes for other arrangements $(\theta \neq \pi / 2)$.

The expression in (29c) has been calculated numerically for three angles of conductivity, $\alpha=5^{\circ}, 45^{\circ}$ and $85^{\circ}$, and for various values of $\theta$ and $(c t / R)$. The plots in Figs. 4(a) - (c) reveal the transition from the monotonic behavior in the $\theta=0$ plane to the peaked response at $(c t / R)=\csc \alpha$ (dashed line) when the observation angle $\theta \rightarrow \pi / 2$. One observes from the different abscissa scales that the fluctuation introduced by the arrival of the surface wave is compressed in time as $\alpha$ increases from small to large values.

The preceding discussion leads to the anticipation that the conventional surface wave in a time-harmonic field turned on suddenly at $t=0$ is not established at a point $y$ on the surface until after a time $t \approx(y / c) \csc \alpha$. This is verified from an examination of the transient solution corresponding to a time function

$$
\begin{array}{ll}
g(t)=0, & t<0, \\
g(t)=\exp (-i \omega t), & t>0,
\end{array}
$$

which (for a source located on the screen) is obtained from (32) and (2b) for $t>y / c$ as

$$
G^{\prime}=\frac{1}{2 \pi} \int_{y / c}^{t}\left[\xi^{2}-(y / c)^{2}\right]^{1 / 2}\left[\xi^{2}-\frac{y^{2} \csc ^{2} \alpha}{c^{2}}\right]^{-1} \exp [-i \omega(t-\xi)] d \xi
$$

or after changing variables to $\zeta=\cosh ^{-1}(\xi c / y)$,

$$
G^{\prime}=\frac{1}{2 \pi} e^{-i \omega t} \int_{0}^{\zeta_{0}} \frac{\sinh ^{2} \zeta \exp [i k y \cosh \zeta]}{\cosh ^{2} \zeta-\csc ^{2} \alpha} d \zeta, \quad \cosh \zeta_{0}=\frac{c t}{y} .
$$

*When $\theta=\pi / 2$, the pole singularities of the integrand in (28) lie on the vertical integration path through the point $w_{1}=w-\pi / 2$. No additional contribution as in (A2) need be considered, however, since $u(\pi / 2-w)$ is in this case an even function of $w$. 
1965]

TRANSIENT SOLUTIONS FOR DIFFRACTION PROBLEMS

163

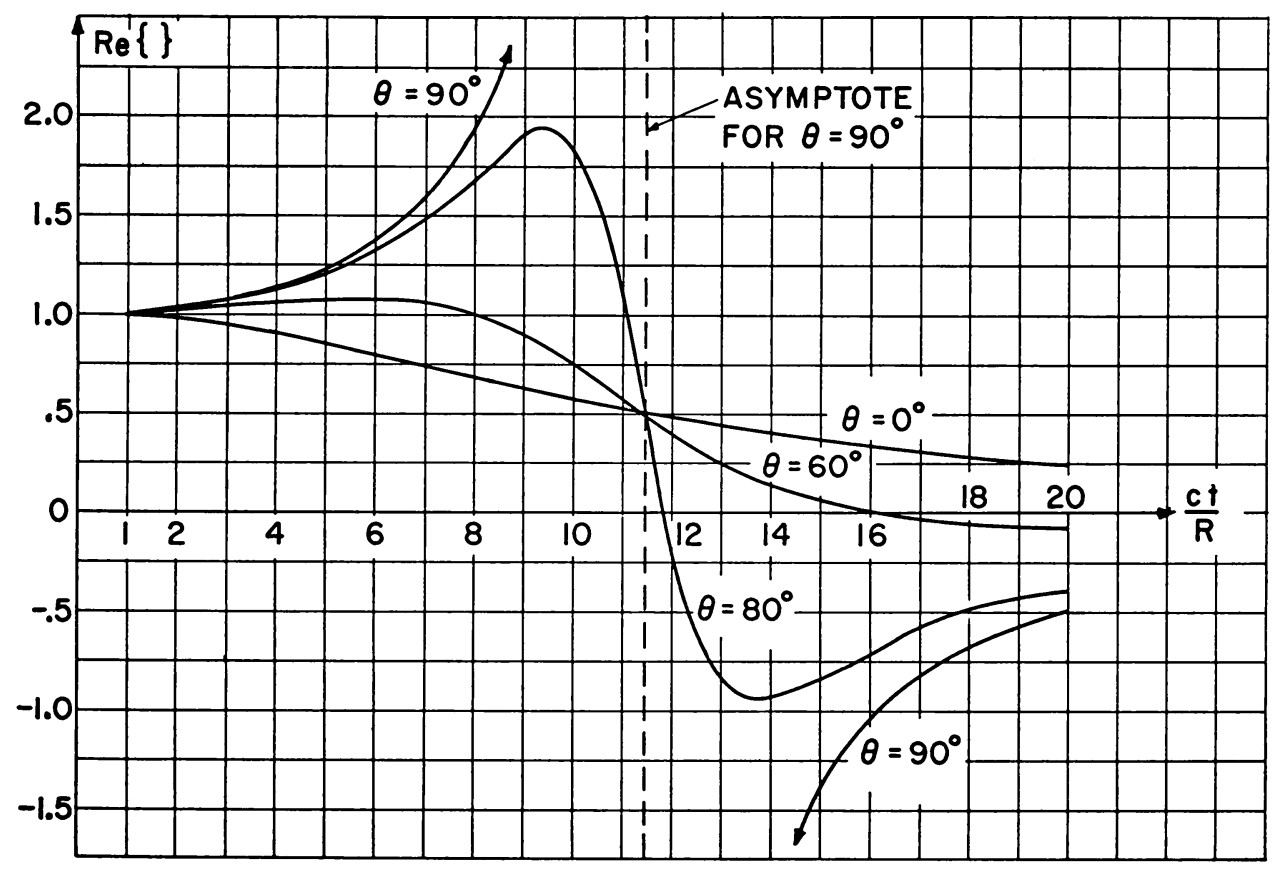

(a) $a=5^{\circ}$

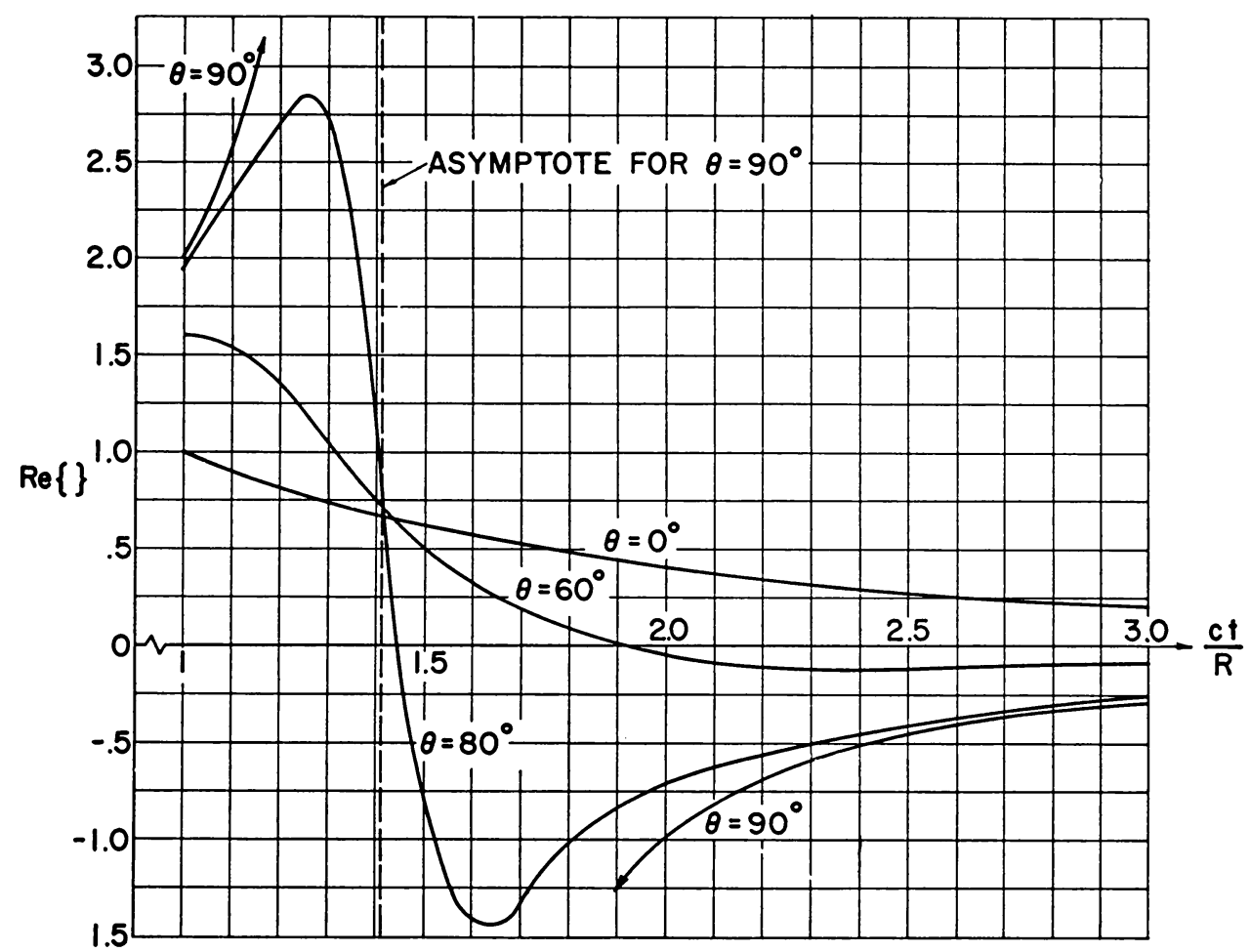

(b) $a=45^{\circ}$ 


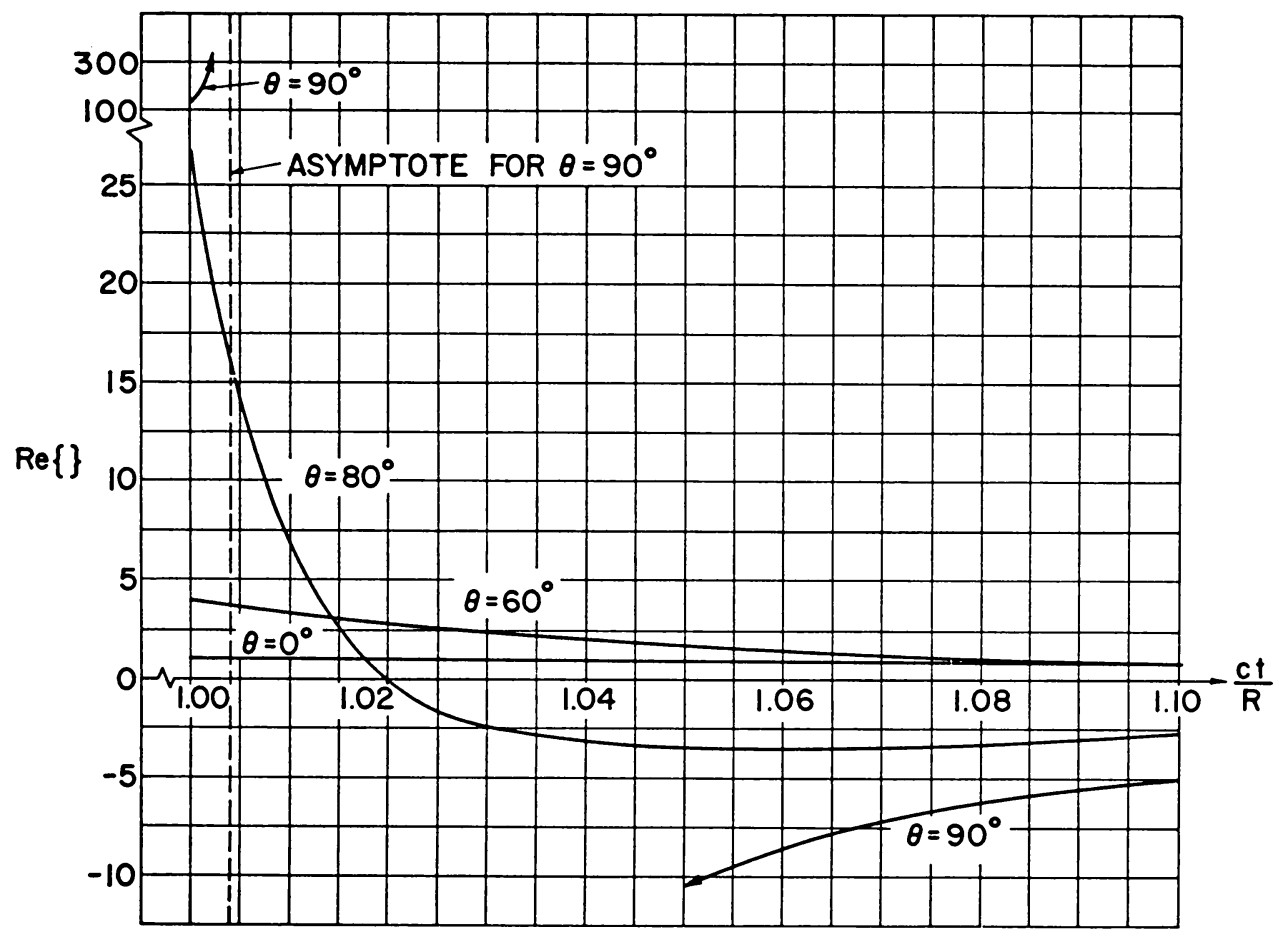

(c) $a=85^{\circ}$

Fig. 4. Plot of the amplitude factor in (29c).
a) $\alpha=5^{\circ}$
b) $\alpha=45^{\circ}$
c) $\alpha=85^{\circ}$

Again, $G^{\prime}=0$ when $t<y / c$. For observation times $t<(y / c) \csc \alpha$, one may expand the denominator in the integrand in a series of powers of $(\cosh \zeta \sin \alpha)$ and each multiplicative factor of $\cosh \zeta$ in the integrand may be replaced by the derivative operator $(i k)^{-1}(d / d y)$. Thus, the integral in (34) may be written as a series involving repeated spatial derivatives of

$$
\int_{0}^{\zeta_{0}} \exp [i k y \cosh \zeta] d \zeta
$$

which expression occurs in the response to a line source in free space. The series converges rapidly if $\left(\cosh \zeta_{0} \sin \alpha\right) \ll 1$ and the resulting field shows little evidence of the presence of a surface wave. However, for $\left(\cosh \zeta_{0} \sin \alpha\right)>1$, one may write the integral in (35) as

$$
\int_{0}^{\zeta_{0}}=\int_{0}^{\infty}-\int_{\zeta_{0}}^{\infty}
$$

with the first contribution on the right-hand side* representing the steady-state solution in $(28)$ or $(28 \mathrm{~b})$ (for $\theta=\pi / 2)$ and the second denoting a correction term. The steady-state

*The integral is taken as the Cauchy principal value with respect to the pole at $\zeta=\cosh ^{-1} \csc ^{*} \alpha$. With an extension of the range of integration from $\zeta=-\infty$ to $\zeta=\infty$ and the change of variable $\zeta=i(w-\pi / 2)$, it is not difficult to effect a reduction to (28). 
response now exhibits clearly the surface wave term (see (28b)), and the correction integral

$I=\int_{\zeta_{0}}^{\infty} \frac{\left(\sinh ^{2} \zeta\right) \exp (i k y \cosh \zeta)}{\cosh ^{2} \zeta-\csc ^{2} \alpha} d \zeta \leq \frac{\left(d^{2} / k^{2} d y^{2}\right)+1}{\csc ^{2} \alpha-\cosh ^{2} \zeta_{0}} \int_{\zeta_{0}}^{\infty} \exp [i k y \cosh \zeta] d \zeta$

is small when $\left(\cosh \zeta_{0} \sin \alpha\right) \gg 1$. Thus, the surface wave appears within a time interval centered about $\cosh \zeta_{0}=\csc \alpha$, as anticipated.

f. Line source and unidirectionally conducting half plane. Seshadri ${ }^{14}$ has also found the field radiated by a line source of electric currents situated on a unidirectionally conducting half plane. If the screen occupies the region $y>0, z=0$, and the line source is located at $y=a, z=0$, as in Fig. 5, the fields may be calculated as before from a potential function $G$ which now has the following form (reference 14, Eq. (61)):

$$
G\left(\mathbf{\varrho}, \mathbf{\varrho}^{\prime} ; \omega\right)=G_{\infty}\left(\mathbf{\varrho}, \mathbf{\varrho}^{\prime} ; \omega\right)+G_{d}\left(\mathbf{\varrho}, \mathbf{\varrho}^{\prime} ; \omega\right), \quad \mathbf{\varrho}^{\prime} \equiv\left(y^{\prime}, z^{\prime}\right)=(a, 0),
$$

where $G_{\infty}\left(\boldsymbol{\varrho}, \mathbf{\varrho}^{\prime} ; \omega\right)$ is the potential function for the infinite screen in the preceding section,

$$
G_{\infty}\left(\boldsymbol{\varrho}, \mathbf{\varrho}^{\prime} ; \omega\right)=\frac{i}{4} H_{0}^{(1)}(k R)-\frac{i \cot ^{2} \alpha}{4 \pi} \int_{P} \frac{\exp [i k R \cos (w-\theta)]}{\csc ^{2} \alpha-\sin ^{2} w} d w,
$$

with $R$ and $\theta$ denoting, respectively, the distance from the source to the observation point $P$ and the angle between $R$ and the positive $z$-axis. $G_{d}\left(\boldsymbol{\varrho}, \mathbf{e}^{\prime} ; \omega\right)$ expresses the perturbation introduced by the terminated screen,

$G_{d}\left(\boldsymbol{\varrho}, \mathbf{\varrho}^{\prime} ; \omega\right)=\frac{i}{4 \pi} \frac{\left(\cot ^{2} \alpha\right) \exp (i k a \csc \alpha)}{(1+\csc \alpha)^{1 / 2}} \int_{P} \frac{(1-\sin w)^{1 / 2} \exp \left[i k R_{1} \cos \left(w-\theta_{1}\right)\right]}{\csc ^{2} \alpha-\sin ^{2} w} d w$

where $R_{1}$ and $\theta_{1}$ are the polar coordinates of the observation point measured from the edge of the screen at $y=z=0$ (see Fig. 5).

The impulse response of the infinite screen is obtained from the Laplace inversion of (38a) and has been discussed in the preceding section. The diffraction field in (38b), apart from the factor $\exp (i k a \csc \alpha)$, is also in the form shown in (1) so that its inversion can be carried out directly. If the time function corresponding to the integral in (38b) is to be denoted by $F(t)$, then the time function corresponding to the integral multiplied by $\exp (i k a \csc \alpha)$ is $F\left(t-t^{\prime}\right)$, where $t^{\prime}=(a / c) \csc \alpha$. From these considerations and the discussion in Section 2 , one obtains for the transient diffraction field,

$$
G_{d}^{\prime}\left(\mathbf{\varrho}, \mathbf{\varrho}^{\prime} ; t\right)=0, \quad t<\frac{R_{1}}{c}+t^{\prime},
$$

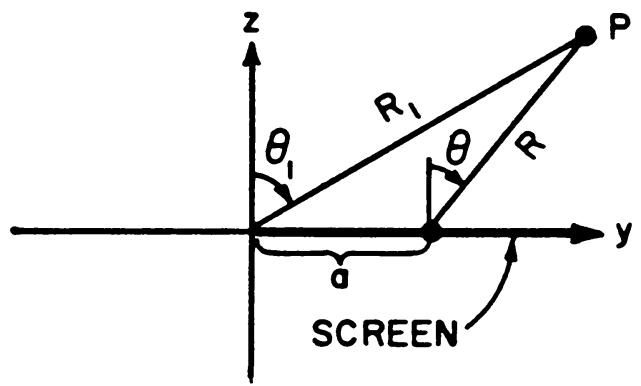

Fig. 5. Line source and unidirectionally conducting half plane. 


$$
\begin{gathered}
G_{d}^{\prime}\left(\boldsymbol{\varrho}, \boldsymbol{\varrho}^{\prime} ; t\right)=\frac{\cot ^{2} \alpha}{\pi[2(1+\csc \alpha)]^{1 / 2}} \frac{\operatorname{Re} Q}{\left[\left(t-t^{\prime}\right)^{2}-\left(R_{1} / c\right)^{2}\right]^{1 / 2}}, \quad t>\frac{R_{1}}{c}+t^{\prime}, \\
Q=\frac{\sin \left\{(1 / 2)\left[(\pi / 2)-\theta_{1}+i \cosh ^{-1}\left(c t / R_{1}-c t^{\prime} / R_{1}\right)\right]\right\}}{\csc ^{2} \alpha-\sin ^{2}\left[\theta_{1}-i \cosh ^{-1}\left(c t / R_{1}-c t^{\prime} / R_{1}\right)\right]},
\end{gathered}
$$

where it has been recognized that $(1-\sin w)^{1 / 2}=2^{1 / 2} \sin \{(\pi / 4)-(w / 2)\}$. Since $t^{\prime}=$ $(a / c) \csc \alpha$ is the time required for the surface wave field to travel from the source point on the screen to the edge, and $\left(R_{1} / c\right)$ is the time of travel of a space wave from the edge to the observation point, one observes that the diffraction field for this source location is excited by the incident surface wave which is then radiated into space.

The region of validity of $(39 \mathrm{~b})$ is confined to observation angles $\left|\theta_{1}\right|<\pi / 2$. When $\left|\theta_{1}\right|=\pi / 2$, the pole singularities in the integrand of (38b) lie on the integration path in (3), and the considerations in the Appendix are appropriate. Since

$$
u(w)=\frac{2^{1 / 2} \sin [(\pi / 4)-(w / 2)]}{\csc ^{2} \alpha-\sin ^{2} w}
$$

one has

$$
u\left(\frac{\pi}{2}+w\right)=\frac{-2^{1 / 2} \sin (w / 2)}{\csc ^{2} \alpha-\sin ^{2} w}, \quad u\left(-\frac{\pi}{2}+w\right)=\frac{2^{1 / 2} \cos (w / 2)}{\csc ^{2} \alpha-\sin ^{2} w},
$$

so that $u\left(\theta_{1}+w\right)$ is an odd function of $w$ when $\theta_{1}=\pi / 2$, but an even function when $\theta_{1}=-\pi / 2$. As the observation point approaches the screen, the diffracted contribution from (39b) tends to zero when $\theta_{1} \rightarrow \pi / 2$ with $c\left(t-t^{\prime}\right) \neq y \csc \alpha$, but becomes infinite when $\theta_{1} \rightarrow \pi / 2$ with $c\left(t-t^{\prime}\right)=R_{1} \csc \alpha$, thereby exhibiting a delta function dependence. The entire diffraction field is therefore contained in the delta function response in (A2), with $w_{1}=\cosh ^{-1} \csc \alpha$ (Note: the integration path is indented into the left half of the $w$-plane to avoid the poles):

$$
G_{d}^{\prime}\left(\boldsymbol{\varrho}, \mathbf{\varrho}^{\prime} ; t\right)=\frac{\cos ^{2} \alpha}{4(1+\sin \alpha)} \delta\left(t-\frac{a+y}{c} \csc \alpha\right), \quad \theta_{1}=\frac{\pi}{2} .
$$

Thus, on the screen, one observes an impulse which arrives at the observation point precisely after the time interval required by the surface waves to travel from the source to the edge and from the edge to the observation point. Unlike the cylindrical diffraction field in space which persists after the arrival of the first response, the diffraction field on the screen maintains the impulsive behavior of the excitation.

When $\theta_{1}=-\pi / 2$, the observation point lies on the portion of the $z=0$ plane which is not occupied by the screen. Since $u(-(\pi / 2)+w)$ is an even function of $w$, the impulse in (A2) is absent and $G_{d}^{\prime}$ is given by the limiting form of (39b),

$$
G_{d}^{\prime}\left(\mathbf{0}, \mathbf{\varrho}^{\prime} ; t\right)=\left\{\begin{aligned}
0, & t<\frac{|y|}{c}+t^{\prime}, \\
\frac{\cot ^{2} \alpha}{\pi[2(1+\csc \alpha)]^{1 / 2}} \frac{c}{2^{1 / 2}|y|}\left[\frac{c\left(t-t^{\prime}\right)}{|y|}-1\right]^{-1 / 2} & \\
\cdot\left[\csc ^{2} \alpha-\left(\frac{c t-c t^{\prime}}{y}\right)^{2}\right]^{-1}, & t>\frac{|y|}{c}+t^{\prime} .
\end{aligned}\right.
$$

with $t^{\prime}=(a / c) \csc \alpha$. To this wave must be added the infinite-plane field contribution from (32), 
$G_{\infty}^{\prime}\left(\mathbf{\varrho}, \mathbf{o}^{\prime} ; t\right)=\frac{1}{2 \pi}\left[t^{2}-\left(\frac{|y|+a}{c}\right)^{2}\right]^{1 / 2}\left[t^{2}-\left(\frac{|y|+a}{c}\right)^{2} \csc ^{2} \alpha\right]^{-1}, \quad t>\frac{|y|+a}{c}$.

While both $G_{d}^{\prime}$ and $G_{\infty}^{\prime}$ have a singularity at $c t=(|y|+a) \csc \alpha$, the time required for the surface wave to travel from the source to the observation point along the infinite screen, one may easily verify that the singularity cancels in the sum $\left(G_{d}^{\prime}+G_{\infty}^{\prime}\right)$. This is in accord with the physical requirement since no surface waves can exist in the region $y<0$.

It is of interest to explore further the different character of the surface waves radiated directly by the line source on the screen (as in (32) and (44)) and the surface waves excited by the edge discontinuity (as in (42)). In the former case, the response is peaked about the time interval $t_{1}$ required by a field traveling at the surface wave speed to cover the distance between the source and observation points; the increase in field strength at a given point $y$ occurs gradually as $t \rightarrow t_{1}$. In (42), on the other hand, the response has the same impulsive dependence as the excitation (see Fig. 6). This behavior may be explained after an examination of the steady-state surface wave fields which are exhibited explicitly by extracting the contributions $G_{\infty s}$ and $G_{d s}$ from the residues at $w_{p}=\pi / 2-i \cosh ^{-1} \csc \alpha$ in $(38 \mathrm{a}, \mathrm{b})$ :

$$
\begin{aligned}
& \left.G_{\infty s}=\frac{i}{4} \cos \alpha \exp (k|i| y-a|\csc \alpha-| z \mid \cot \alpha]\right), \quad k=\frac{\omega}{c}, \\
& G_{d s}=\frac{1}{4} \cos \alpha\left[\frac{\csc \alpha-1}{\csc \alpha+1}\right]^{1 / 2} \exp (k[i(y+a) \csc \alpha-|z| \cot \alpha]) .
\end{aligned}
$$

While these results differ in the $k$-dependent phase terms involving $|y-a|$ and $(y+a)$, respectively, and also by the real amplitude coefficient $(\csc \alpha-1)^{1 / 2}(\csc \alpha+1)^{-1 / 2}$, the significant difference between the two expressions is in the presence of the phase factor $i=\exp (i \pi / 2)$ in (45a).

To find the real, time-dependent surface wave fields $G_{s}^{\prime}$ excited by an impulsive source distribution $\delta(t)$, it is convenient to employ the inverse Fourier transform

$$
G_{s}^{\prime}=\frac{1}{2 \pi} \int_{-\infty}^{\infty} G_{s} e^{-i \omega t} d \omega=\frac{1}{2 \pi}\left[\int_{0}^{\infty} G_{s} e^{-i \omega t} d \omega+\int_{0}^{\infty} G_{s}^{*} e^{i \omega t} d \omega\right],
$$

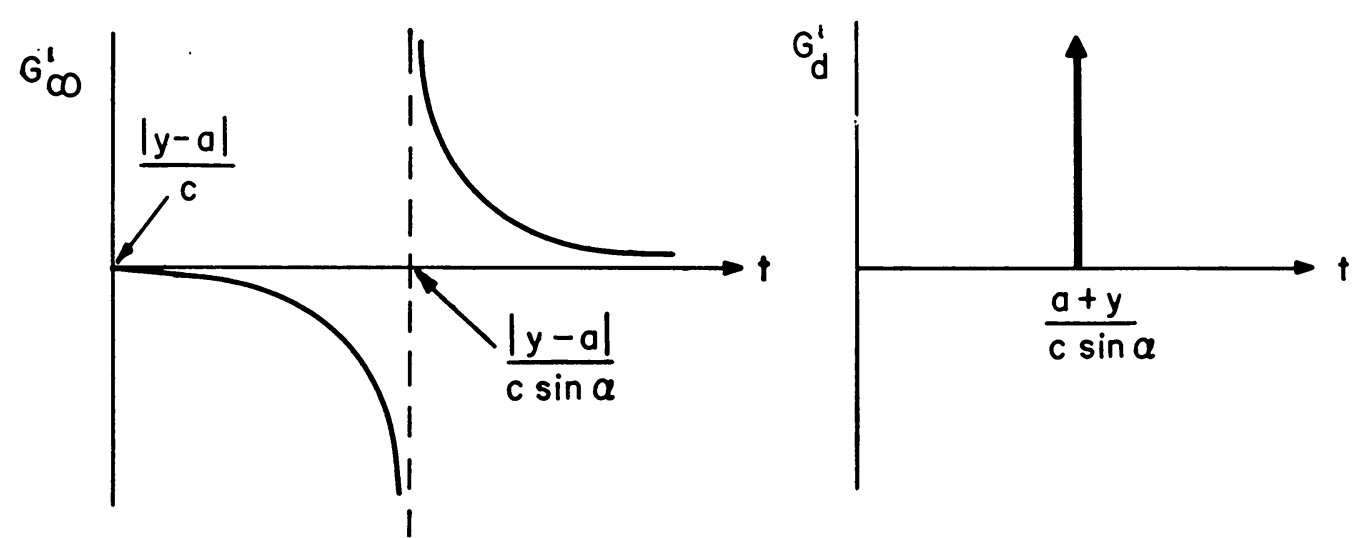

Fig. 6. Field behavior on the screen.
a) due to line source
b) due to edge 
which is most useful in the second form wherein the integration extends only over positive frequencies. One easily obtains the results,

$$
\begin{aligned}
& G_{\infty s}^{\prime}=-\frac{\cos \alpha}{4 \pi}\left(\frac{|y-a|}{c} \csc \alpha-t\right)\left[\left(\frac{|y-a|}{c} \csc \alpha-t\right)^{2}+\left(\frac{|z|}{c} \cot \alpha\right)^{2}\right]^{-1}, \\
& G_{d s}^{\prime}=\frac{\cos \alpha}{4 \pi}\left(\frac{\csc \alpha-1}{\csc \alpha+1}\right)^{1 / 2} \frac{|z|}{c} \cot \alpha\left[\left(\frac{y+a}{c} \csc \alpha-t\right)^{2}+\left(\frac{|z|}{c} \cot \alpha\right)^{2}\right]^{-1},
\end{aligned}
$$

which do not behave identically as $z \rightarrow 0$. The line-source excited contribution varies on the screen according to

$$
G_{\infty s}^{\prime}=(-\cos \alpha)\left[4 \pi\left(\frac{|y-a|}{c} \csc \alpha-t\right)\right]^{-1}, \quad|z|=0,
$$

while the diffracted contribution vanishes unless $c t=(y+a) \csc \alpha$. This limiting behavior may be formulated in terms of the delta function, ${ }^{15}$

$$
G_{d s}^{\prime}=\frac{\cos \alpha}{4}\left[\frac{\csc \alpha-1}{\csc \alpha+1}\right]^{1 / 2} \delta\left(t-\frac{y+a}{c} \csc \alpha\right), \quad|z|=0,
$$

and yields the same expression as in (42). Thus, the phase factor $i$ in (45a) serves to smear out the response to the impulsive excitation, whereas its form is retained for (45b).

4. Summary. It has been shown how a class of integral solutions for time-harmonic diffraction problems may be inverted in an elementary fashion to yield the response to a temporal impulse. Various illustrative examples have been studied, including the diffraction of a cylindrical pulse by a dielectric half space, a perfectly absorbing and perfectly reflecting wedge, and a unidirectionally conducting infinite and semi-infinite screen. The latter configuration is of particular interest since it may support surface waves which are excited either by the source or by the edge discontinuity. In contrast to the time-harmonic solution, the transient surface wave fields have different characteristics for these two excitation mechanisms.

\section{Appendix}

The effect of pole singularities on the integration path. If $u(w+\varphi)$ in (3) has pole singularities on the imaginary axis in the $w$-plane, the inversion procedure must be modified. Of pertinence to the problems discussed in the text is the case where $u(w+\varphi)$ has simple poles at $w= \pm i w_{1}, w_{1}$ positive real, with the integration path indented around the poles into the half plane $\operatorname{Re} w<0$. One obtains then instead of (5),

$$
\begin{aligned}
G(\gamma, \varphi ; i s) & =-i a\left(\frac{i s}{c}\right) P \int_{\gamma / c}^{\infty}\left[\tau^{2}-\left(\frac{\gamma}{c}\right)^{2}\right]^{-1 / 2}\left\{u\left[\varphi-i \cosh ^{-1}\left(\frac{c \tau}{\gamma}\right)\right]\right. \\
& \left.+u\left[\varphi+i \cosh ^{-1}\left(\frac{c \tau}{\gamma}\right)\right]\right\} \exp (-s \tau) d \gamma+a\left(\frac{i s}{c}\right) \pi i \exp \left[-s(\gamma / c) \cosh w_{1}\right] \\
& \cdot\left\{\left(w-i w_{1}\right)[u(\varphi+w)-u(\varphi-w)]\right\}_{w=i w_{2}},
\end{aligned}
$$

where $P$ denotes the principal value of the integral with respect to the pole singularities, and the second term comprises the half residues arising from the semi-circular path segments around the poles. The inverse Laplace transform of the integral in (A1) yields 
the same result as in $(7 \mathrm{a}, \mathrm{b})$, while the residue contributions yield an additional delta function (note: $e^{-8 \psi}=\int_{0}^{\infty} e^{-8 \tau} \delta(\tau-\psi) d \tau, \psi>0$ ):

$\left.G^{\prime}(\gamma, \varphi ; t)\right|_{\text {res }}=\pi i a\left(\frac{i}{c} \frac{d}{d t}\right)\left\{\left(w-i w_{1}\right)[u(\varphi+w)-u(\varphi-w)]\right\}_{w=i w_{1}} \delta\left(t-\frac{\gamma}{c} \cosh w_{1}\right)$.

If the integration path in (3) avoids the poles through an indentation into the half plane $\operatorname{Re} w>0$, the result in (A2) must be multiplied by $(-1)$.

One observes that the residue contribution vanishes when $u(\varphi+w)$ is an even function of $w$.

\section{REFERENCES}

1. J. B. Keller and A. A. Blank, Diffraction and reflection of pulses by wedges and corners, Comm. Pure and App. Math. 4 (1951) 75

2. H. Bremmer, The pulse solution connected with the Sommerfeld problem for a dipole in the interface between two dielectrics, Proceedings of a Symposium on Electromagnetic Waves, University of Wisconsin Press, Madison, 1962, p. 39

3. M. Papadoponlos, The refraction of a spherical pulse at a plane interface, Report No. 279, Dec. 1961; Diffraction of pulses by a half plane. I, Report No. 293, Feb. 1962. U. S. Army Mathematics Research Center, University of Wisconsin, Madison, Wis.

4. F. G. Friedlander, Sound pulses, Cambridge University Press, 1958

5. L. Cagniard, Reflection and refraction of progressive seismic waves, translated by E. Flinn and C. H. Dix, McGraw-Hill, New York, 1962

6a. C. L. Pekeris, Solution of an integral equation occurring in impulsive wave propagation problems, Proc. Nat. Acad. Sci. 42 (1956), 439 (references to earlier articles by the same author are also given here)

b. C. L. Pekeris and H. Lifson, Motion of the surface of a uniform elastic half space produced by a buried pulse, J. Acoust. Soc. Amer. 29 (1957) 1233

c. C. L. Pekeris and Z. Alterman, Radiation resulting from an impulsive current in a vertical antenna placed on a dielectric ground, J. App. Physics 28 (1957) 1317

7a. A. T. de Hoop, A modification of Cagniard's method for solving seismic pulse problems, App. Sci. Res., Sec. B, 8 (1960) 349

b. A. T. de Hoop and H. J. Frankena, Radiation of pulses generated by a vertical electric dipole above a plane, non-conducting earth, App. Sci. Res., Sec. B, 8 (1960) 369

c. A. T. de Hoop, Representation theorems for the displacement in an elastic solid and their application to elastodynamic diffraction theory, Dissertation, University of Delft, Netherlands, 1958

8. B. van der Pol and A. H. M. Levelt, On the propagation of a discontinuous electromagnetic wave, Koninkl. Nederl. Akad. v. Wetenschappen, Amsterdam, 63 (1960) 254

9. cf. M. Gardner and J. Barnes, Transients in linear systems, John Wiley and Sons, 1942. Vol. I, Ch. 5

10. cf. L. B. Felsen and N. Marcuvitz, Modal analysis and synthesis of electromagnetic fields, Microwave Research Institute, Polytechnic Institute of Brooklyn, Report PIBMRI-841-60, Ch. 5, Sec. C3

11. A. Sommerfeld, Mathematische Theorie der Diffraktion, Math. Ann. 47 (1896) 317

12. reference 10 , Chapter 6 , Sec. D

13. F. Oberhettinger, On the diffraction and reflection of waves and pulses by wedges and corners, J. Research (NBS) 61 (1958) 343 (an extensive list of references is contained in this paper)

14. S. R. Seshadri, Excitation of surface waves on a unidirectionally conducting screen, IRE Transactions Microwave Theory and Techniques, MTT'-10 (1962) 279

15. cf. P. M. Morse and H. Feshbach, Methods of theoretical physics, McGraw-Hill Book Co., New York, 1953, p. $813-814$ 